\title{
Palladium-catalysed Regioselective Cyclisation of Unsaturated Bromoanilinoalkenenitriles
}

\author{
Chau-Chen Yang, * a Pei-Jiun Sun ${ }^{a}$ and Jim-Min Fang ${ }^{b}$ \\ a Department of Cosmetic Science, Chia Nan Junior College of Pharmacy, Tainan 717, Taiwan, Republic of China \\ b Department of Chemistry, National Taiwan University, Taipei 107, Taiwan, Republic of China
}

$\alpha$-(o-Bromoanilino)alkenenitriles are converted to 2-(methylamino)benzonitrile and varied $\gamma$-carbolines by catalysis with palladium; the reactions may involve intramolecular aryl additions to the cyano group and other processes such as cyano group transposition, electrocyclisation, ethyl group transfer and oxidative aromatisation.

Heck reactions are important palladium-catalysed reactions for carbon-carbon bond formation such as the well documented arylation of olefins $; 1$ the corresponding arylation of a cyano group is, however, not reported. Our investigation of the palladium-catalysed cyclisations of varied $\alpha$ - $(o$-bromoanilino)alkenenitriles 1 and 2 showed that the reactions occurred exclusively at the cyano group to give 2-(methylamino)benzonitrile $^{2} 3$ and $\gamma$-carbolines ${ }^{3} 4$ and 5 (Scheme 1). Non-conjugated alkenenitriles 1a-c were prepared, ${ }^{4}$ by condensation of equimolar amounts of an appropriate unsaturated aldehyde, potassium cyanide, $N$-methyl-o-bromoaniline ${ }^{5}$ and hydrochloric acid $\left(12 \mathrm{~mol} \mathrm{dm}^{-3}\right)$. By a similar procedure, $1 \mathbf{d}$ was prepared in $78 \%$ yield from cyclopropanecarbaldehyde. Treatment of $1 \mathrm{a}-\mathrm{c}$ with the strong base $\mathrm{Bu}$ tOK in $\mathrm{Bu}^{\mathrm{t} O H}-$ THF at $0{ }^{\circ} \mathrm{C}$ gave the more stable conjugated $\alpha$-amino alkenenitriles $2 \mathbf{a}-\mathbf{c}$ having the $2 E$-configuration. ${ }^{6}$ In a typical

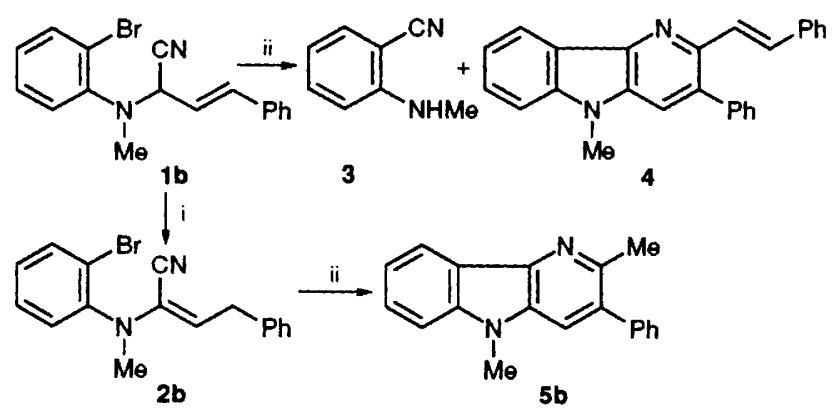

Scheme 1 Reagents and conditions: $\mathrm{i}, \mathrm{Bu} \mathrm{t}^{\mathrm{O}}$ ( 1 equiv.), $\mathrm{Bu} \mathrm{t} \mathrm{OH}-\mathrm{THF}$, $0{ }^{\circ} \mathrm{C}, 1 \mathrm{~h} ; \mathrm{ii}, \mathrm{Pd}(\mathrm{OAc})_{2}, \mathrm{Ph}_{3} \mathrm{P}, \mathrm{Et}_{3} \mathrm{~N}, \mathrm{DMF}, 100{ }^{\circ} \mathrm{C}, 6 \mathrm{~h}$

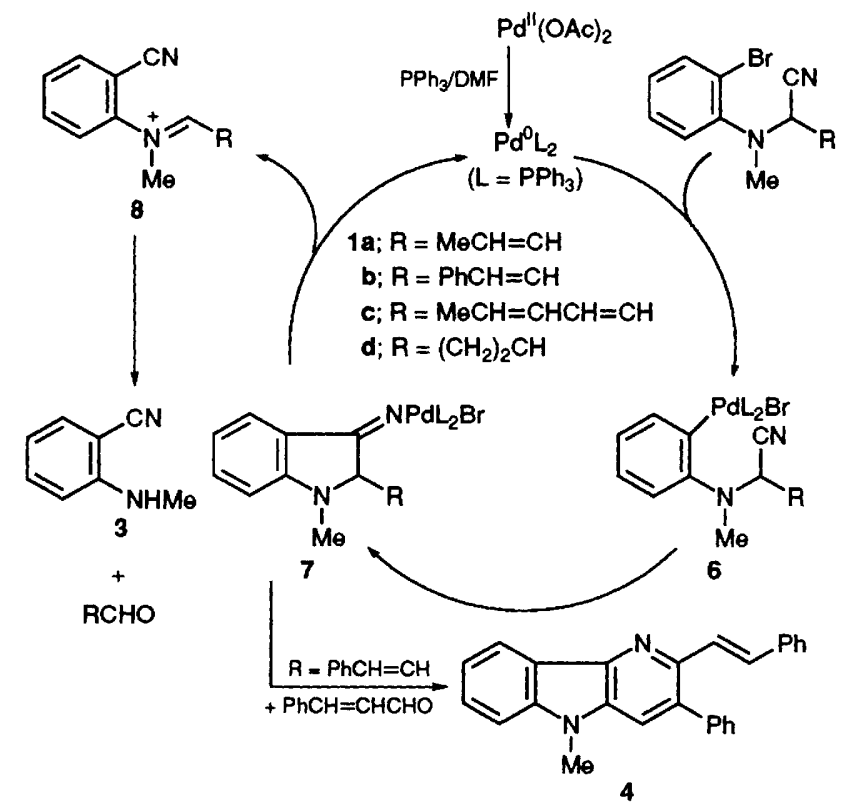

Scheme 2 procedure, $\mathbf{1 b}(1 \mathrm{mmol})$ in DMF $(15 \mathrm{ml})$ was treated with $\mathrm{Pd}(\mathrm{OAc})_{2}(0.1 \mathrm{mmol}), \mathrm{Ph}_{3} \mathrm{P}(0.2 \mathrm{mmol})$ and $\mathrm{Et}_{3} \mathrm{~N}(1.2 \mathrm{mmol})$ for $6 \mathrm{~h}$ at $100{ }^{\circ} \mathrm{C}$ under an argon atmosphere. ${ }^{7}$ The reaction mixture was cooled and EtOAc $(30 \mathrm{ml})$ was added. The mixture was washed with water, the organic phase was concentrated and chromatographed on a silica-gel column by elution with gradients of EtOAc-hexane to give 2-(methylamino)benzonitrile 3 and $\gamma$-carboline 4 in 38 and $36 \%$ yields, respectively. Benzonitrile 3 was also obtained in $72-85 \%$ yields from the reactions of $\mathbf{1 a}, \mathbf{1 c}$ and $\mathbf{1 d}$. The palladiumcatalysed reactions of $\mathbf{2 a}-\mathbf{c}$ afforded $\gamma$-carbolines $\mathbf{5 a - c}$ in $75-88 \%$ yields. The structures of 4 and 5 were rigorously determined. $\dagger$

The mechanisms for the present palladium-catalysed reactions are not fully understood. The reactions of $\mathbf{1 a - d}$ are presumably initiated by oxidative insertion of $\mathrm{Pd}^{0}$ to the bromophenyl groups (Scheme 2). The organopalladium 6 undergoes cyclisation by attacking at the cyano group, giving 7 , but not at the olefinic double bonds. The iminoindoline 7 may rupture to an iminium ion $\mathbf{8}$, which is subsequently hydrolysed to give 2-(methylamino) benzonitrile and aldehydes RCHO. When $\mathrm{R}$ is a styryl group, 7 may react further with cinnamaldehyde to give $\gamma$-carboline 4. Compounds $2 a-c$ also undergo oxidative insertions with $\mathrm{Pd}^{0}$, giving the intermediates 9 . For $2 c$ ( $R$ is a butenyl group), a $6 \pi$ electrocyclisation and subsequent elimination of hydridopalladium produce $\gamma$-carboline 5c. Though the intermediate 9 derived from $2 \mathbf{a}, \mathbf{b}$ ( $\mathrm{R}=\mathrm{Et}$ or $\mathrm{PhCH}_{2}$ ) cannot undergo electrocyclisation, it may take an ethyl group from $\mathrm{Et}_{3} \mathrm{NH}^{+}$, giving 10. Subsequent elimination of hydridopalladium, electrocyclisation and oxidative aromatisation would furnish the $\gamma$-carbolines $\mathbf{5 a , b}$ (Scheme 3).

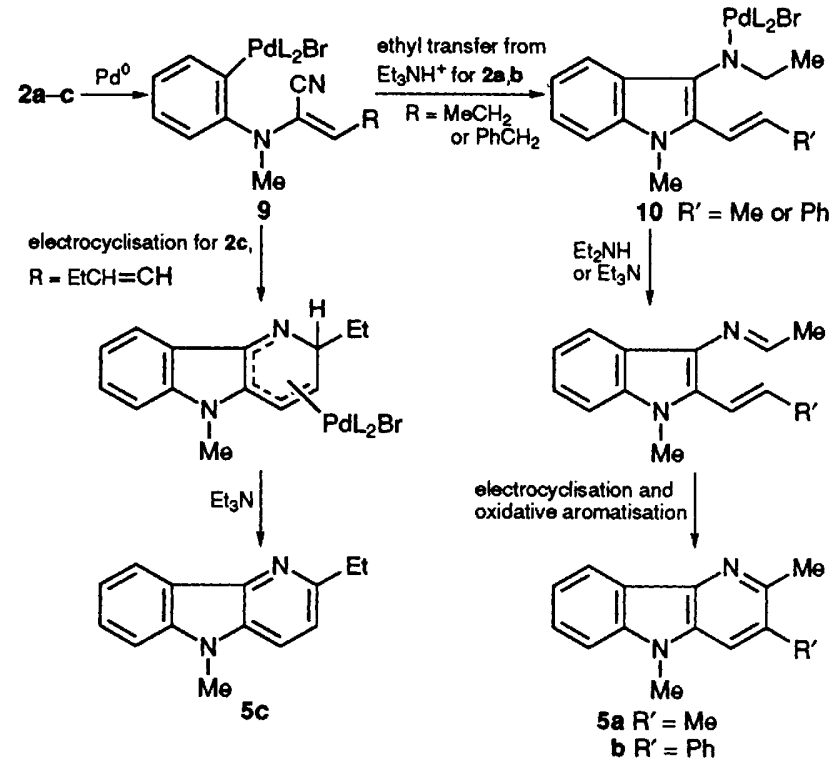

Scheme 3 
Our study of the palladium-catalysed reactions shows three uncommon features: (i) transposition of the cyano group in conversion of 1a-d to $\mathbf{3}$, (ii) two covalent bonds are formed in coversion of $\mathbf{2 c}$ to $\mathbf{5 c}$ by consecutive aryl-cyano addition and electrocyclisation, and (iii) involvement of an ethyl group transfer in conversion of $\mathbf{2 a}, \mathbf{b}$ to $\mathbf{5 a}, \mathbf{b}$

We thank the National Science Council of the Republic of China for financial support (Grant NSC82-0208-M041-006).

Received, 1st September 1994; Com. 4/05346J

\section{Footnote}

+ All new compounds gave satisfactory spectral and analytical data.

\section{References}

1 R. F. Heck and M. Terpko, J. Am. Chem. Soc., 1979, 101, 5281; R. F. Heck, Org. React., 1982, 27. 345; R. F. Heck, Pure Appl. Chem., 1981, 53, 2323; G. D. Davis Jr. and A. Hallberg, Chem. Rev. 1989, 89, 1433.

2 A. Couture, E. Deniau, Y. Gimbert and P. Grandclaudon, Tetrahedron, 1993, 49, 1431.

3 R. A. Abramovitch, Can. J. Chem., 1960, 38, 2271.

4 J.-M. Fang, L.-F. Liao and C.-C. Yang, Proc. Natl. Sci. Council (Taipei), 1985, 9, 1; J.-M. Fang, C.-C. Yang and Y.-W. Wang, J. Org. Chem., 1989, 54, 477; J.-M. Fang, C.-C. Yang and Y.-W. Wang, J. Org. Chem. . 1989, 54, 481.

5 S. B. Kadin, J. Org. Chem., 1973, 38, 1348.

6 J.-M. Fang and H.-T. Chang, J. Chem. Soc., Perkin Trans. I, 1988 , 1945; J.-M. Fang and C.-J. Chang, J. Chem. Soc., Chem. Commun., 1989, 1787.

7 W. Cabri, H. Candiani and A. Bedeschi, J. Org. Chem., 1992, 57, 3558. 